\title{
MULTIPLICITY RESULTS FOR SOME NONLINEAR ELLIPTIC PROBLEMS
}

\author{
KUAN-JU CHEN
}

(Received 2 October 2002; revised 13 November 2002)

\author{
Communicated by K. Wysocki
}

\begin{abstract}
In this paper, first, we study the existence of the positive solutions of the nonlinear elliptic equations in unbounded domains. The existence is affected by the properties of the geometry and the topology of the domain. We assert that if there exists a $(P S)_{c}$-sequence with $c$ belonging to a suitable interval depending by the equation, then a ground state solution and a positive higher energy solution exist, too. Next, we study the upper half strip with a hole. In this case, the ground state solution does not exist, however there exists at least a positive higher energy solution.
\end{abstract}

2000 Mathematics subject classification: primary 35J20, 35J25.

Keywords and phrases: Palais-Smale sequence, higher energy solution, upper half strip with a hole.

\section{Introduction}

In this paper we study the following problem:

$$
\begin{cases}-\Delta u+u=f(x, u) & \text { in } \Omega, \\ u>0 & \text { in } \Omega, \\ u \in H_{0}^{1}(\Omega), & \end{cases}
$$

where $\Omega \subset \mathbb{R}^{N}$ is an unbounded domain with smooth boundary $\partial \Omega, f: \vec{\Omega} \times \mathbb{R} \rightarrow \mathbb{R}$ is of class $C^{1}$ and satisfies the following conditions:

(f1) $f(x, y)=o(y)$ near $y=0$ uniformly in $x \in \bar{\Omega}$.

(f2) There exists $a_{1}>0$ such that $\left|f_{y}(x, y)\right| \leq a_{1}\left(1+|y|^{p-1}\right)$ for all $x \in \bar{\Omega}$ and $y \in \mathbb{R}$, where $1<p<(N+2) /(N-2)$ if $N>2$ and $1<p<\infty$ if $N=1,2$.

(C) 2004 Australian Mathematical Society 1446-7887/04 \$A2.00+0.00 
(f3) There exists $\theta>2$ such that $0<\theta F(x, y) \leq f(x, y) y$ for all $x \in \bar{\Omega}$ and $y \in \mathbb{R} \backslash\{0\}$, where $F(x, y)=\int_{0}^{y} f(x, \tau) d \tau$.

(f4) $f(x, t y) y / t$ is a strictly increasing function of $t>0$ for all $x \in \bar{\Omega}$ and $y \in \mathbb{R} \backslash\{0\}$.

Since we will only look for positive solutions of (1.1), it is convenient to define $f(x, u) \equiv 0$ for $u \leq 0$ and $x \in \bar{\Omega}$.

Associated with the problem (1.1) is the energy functional $I$ defined by

$$
I(u)=\frac{1}{2} \int_{\Omega}\left(|\nabla u|^{2}+u^{2}\right) d x-\int_{\Omega} F(x, u) d x .
$$

And $I \in C^{1}\left(H_{0}^{1}(\Omega), \mathbb{R}\right)$.

It is well known that the solutions of (1.1) are the critical points of the energy functional $I$. Moreover, standard arguments from elliptic regularity theory show that critical points of $I$ on $H_{0}^{1}(\Omega)$ are classical solutions of (1.1). However, when $\Omega$ is an unbounded domain the existence of a solution becomes a difficult problem, due to the embedding $j: H_{0}^{1}(\Omega) \rightarrow L^{p}(\Omega)$ which is not compact. The lack of compactness implies that $l$ does not satisfy the Palais-Smale condition, and the standard variational techniques cannot be applied to this problem, so new analyses are needed to solve such problems.

Denote by

$$
\begin{aligned}
M(\Omega) & =\left\{u \in H_{0}^{1}(\Omega) \backslash\{0\} \mid \int_{\Omega}\left(|\nabla u|^{2}+u^{2}\right) d x=\int_{\Omega} f(x, u) u d x\right\}, \\
\alpha_{M}(\Omega) & =\inf _{u \in M(\Omega)} I(u) .
\end{aligned}
$$

Looking for solutions of (1.1) is equivalent to find critical points of $I$ constrained to lie upon the manifold $M(\Omega)$. As a consequence of Ekeland's variational principle, there exists a sequence $\left\{u_{k}\right\} \subset M(\Omega)$ such that

$$
I\left(u_{k}\right) \rightarrow \alpha_{M}(\Omega), \quad I^{\prime}\left(u_{k}\right) \rightarrow 0 \quad \text { in } H^{-1}(\Omega) .
$$

Although $\alpha_{M}(\Omega)$ does not guarantee the existence of a critical point $u \in H_{0}^{1}(\Omega)$ with $I(u)=\alpha_{M}(\Omega)$, we can analyze Palais-Smale sequences to justify whether there exist positive solutions of (1.1) or not. New analysis is needed for solving such problems which will be described as follows. Let

$$
\begin{aligned}
& \Omega_{k}=\Omega \cap B_{k}^{N}(0) ; \quad \text { where } \quad B_{k}^{N}(0)=\left\{x \in \mathbb{R}^{N} \mid\|x\|<k\right\}, \\
& \widetilde{\Omega}_{k}=\Omega \backslash \overline{B_{k}^{N}(0)} .
\end{aligned}
$$

For $v \in H_{0}^{1}\left(\widetilde{\Omega}_{k+1}\right)$, it can be identified with an element of $H_{0}^{1}\left(\widetilde{\Omega}_{k}\right)$ by extending $v$ to be zero in $\widetilde{\Omega}_{k} \backslash \widetilde{\Omega}_{k+1}$.

In the following definitions, we abbreviate Palais-Smale by (PS). 
DEFINITION 1.1. (1) For $c \in \mathbb{R}$, a sequence $\left\{u_{k}\right\} \subset H_{0}^{1}(\Omega)$ is a $(P S)_{c}$-sequence if $I\left(u_{k}\right) \rightarrow c$ and $I^{\prime}\left(u_{k}\right) \rightarrow 0$ in $H^{-1}(\Omega)$;

(2) $c \in \mathbb{R}$ is a $(P S)$-value if there exists a $(P S)_{c}$-sequence;

(3) I satisfies the $(P S)_{c}$-condition if every $(P S)_{c}$-sequence for $I$ contains a convergent subsequence.

This paper is organized as follows. In Section $2, \Theta(\Omega)$ is the set of all the positive $(P S)$-values. In particular, $\Theta(\Omega)$ contains all the positive critical values of $I$. Let $\delta(\Omega)$ be the infimum of $\Theta(\Omega)$, it will be shown that $\Theta(\Omega)$ is a nonempty set, $\delta(\Omega)$ is a positive number, and the optimal lower bound for $\Theta(\Omega)$ is $\alpha_{M}(\Omega)$ when (f1)-(f4) are satisfied, that is, $\delta(\Omega)=\alpha_{M}(\Omega)$.

If $u$ is a nontrivial solution of (1.1), then multiplying (1.1) by $u$ and integrating by parts shows $u \in M(\Omega)$. For any $u \in H_{0}^{1}(\Omega) \backslash\{0\}$ and $t>0$, let $h_{u}(t)=I(t u)$. By (f1)-(f3), we have $h_{u}(0)=0, h_{u}(t)>0$ for $t$ small, and $h_{u}(t)<0$ for $t$ large. So $u=0$ is a local minimum but not a global minimum of $I$. Therefore, $\max _{t \geq 0} h_{u}(t)$ exists and is achieved at $t_{u}>0$, we get

$$
h_{u}^{\prime}\left(t_{u}\right)=0=t_{u}\|u\|_{H^{1}(\Omega)}^{2}-\int_{\Omega} f\left(x, t_{u} u\right) u d x
$$

which implies $t_{u} u \in M(\Omega)$. Moreover by (f4), $t_{u}$ is the unique value of $t>0$ such that $t_{u} u \in M(\Omega)$. This implies $M(\Omega)$ is radially homeomorphic to the unit ball in $H_{0}^{1}(\Omega)$.

In Section 3, we assert that if $\alpha_{M}(\Omega)<\alpha_{M}\left(\widetilde{\Omega}_{k}\right)$ for some large $k \in \mathbb{N}$, then there exists a ground state solution $u$ of $(1.1)$ with $I(u)=\alpha_{M}(\Omega)$. And if there exists a $(P S)_{c}$-sequence with $\alpha_{M}(\Omega)<c<\alpha_{M}\left(\widetilde{\Omega}_{k}\right)$ for some large $k \in \mathbb{N}$, then there exist at least two positive solutions of (1.1), that is, a ground state solution $u$ and a positive higher energy solution.

In Section 4, we describe the (PS)-conditions and give a necessary and sufficient conditions in $\Omega$ for which $I$ satisfies the $(P S)_{\alpha_{M}(\Omega)}$-condition.

In the final section, the domain is the upper half strip with a hole. For simplicity, we consider the case where $f$ does not depend on $x$, so the problem is as follows:

$$
\begin{cases}-\Delta u+u=f(u) & \text { in } \Lambda, \\ u>0 & \text { in } \Lambda, \\ u \in H_{0}^{1}(\Lambda), & \end{cases}
$$

where $\Lambda$ is the upper half strip with a hole. Denote by

$$
\begin{aligned}
& A^{r}=\left\{(\xi, \eta) \in \mathbb{R}^{N-1} \times \mathbb{R}|| \xi \mid<r\right\}, \\
& A_{a}^{r}=\left\{(\xi, \eta) \in A^{r} \mid \eta>a\right\}, \quad \text { where } a \in \mathbb{R} .
\end{aligned}
$$


For the strip domain $A^{r}$, Chen [2] modified the results of Lions [6] and asserted that there exists a ground state solution of (1.2) if $\Lambda=A^{r}$. Chen-Chen-Wang [3] established its asymptotic behaviour and showed that the solution is spherically symmetric in $\xi$ and axially symmetric in $\eta$.

For the upper half strip domain $A_{0}^{r}$ (an Esteban-Lions domain), Esteban-Lions [5] asserted that there does not exist any solution of (1.2) if $\Lambda$ is an Esteban-Lions domain. The Esteban-Lions domain is defined as follows:

DEFINITION 1.2. $\Omega \subset \mathbb{R}^{N}$ is an Esteban-Lions domain if there exists $\chi \in \mathbb{R}^{N}$, $\|\chi\|=1$ such that $n(x) \cdot \chi \geq 0$, and $n(x) \cdot \chi \not \equiv 0$ on $\partial \Omega$, where $n(x)$ denotes the unit outward normal to $\partial \Omega$ at the point $x$.

An interesting question is whether there exists a positive higher energy solution of (1.2) if $\Lambda$ is an Esteban-Lions domain with a hole. The question seems to be quite challenging and hard to give a complete answer. We had known that Pohozaev [7] proved that the Dirichlet problem $-\Delta u+u^{(N+2) /(N-2)}=0$ in a ball does not have any nontrivial solution, but Coron [4] proved that there exists a positive solution if we take some small ball out. When $f(u)=u^{p}$, Benci-Cerami [1] asserted that problem (1.2), if $\Lambda$ is an exterior domain, admits a positive higher energy solution. We use a new method, different from Benci-Cerami [1], to prove that if there exists a $(P S)_{c}$-sequence with $c>0$ and $c \notin \Theta\left(\tilde{\Lambda}_{m}\right)$ for some $m \in \mathbb{N}$, then there exists at least a positive higher energy solution of (1.2) if $\Lambda$ is the upper half strip with a hole.

\section{The (PS)-value}

We will introduce some preliminaries to analyze the behaviour of Palais-Smale sequence and study the set $\Theta(\Omega)$ of all the positive (PS)-values.

LEMMA 2.1. If $\left\{u_{k}\right\}$ is a (PS) $)_{c}$-sequence, then there exists a constant $\bar{c}>0$ such that $\left\|u_{k}\right\|_{H^{\prime}(\Omega)} \leq \bar{c}$ for each $k$, and $c \geq 0$. If $c>0$, then there exist a subsequence, still denoted by $\left\{u_{k}\right\}$, a constant $c^{\prime}>0$, such that $\left\|u_{k}\right\|_{H^{\prime}(\Omega)} \geq c^{\prime}$.

Proof. By (f3) and if $k$ is large, then

$$
\begin{aligned}
c+o(1)\left(1+\left\|u_{k}\right\|_{H^{\prime}(\Omega)}\right) & =I\left(u_{k}\right)-\frac{1}{\theta}\left(I^{\prime}\left(u_{k}\right), u_{k}\right) \\
& =\left(\frac{1}{2}-\frac{1}{\theta}\right)\left\|u_{k}\right\|_{H^{\prime}(\Omega)}^{2}-\int_{\Omega}\left[F\left(x, u_{k}\right)-\frac{1}{\theta} f\left(x, u_{k}\right) u_{k}\right] d x \\
& \geq\left(\frac{1}{2}-\frac{1}{\theta}\right)\left\|u_{k}\right\|_{H^{\prime}(\Omega)}^{2} .
\end{aligned}
$$


Thus $\left\|u_{k}\right\|_{H^{1}(\Omega)} \leq \bar{c}$ for each $k$. Then for large $k$, we have $\left(I^{\prime}\left(u_{k}\right), u_{k}\right)=o(1)$ and

$$
c+o(1)=I\left(u_{k}\right)-\frac{1}{\theta}\left(I^{\prime}\left(u_{k}\right), u_{k}\right) \geq\left(\frac{1}{2}-\frac{1}{\theta}\right)\left\|u_{k}\right\|_{H^{\prime}(\Omega)}^{2},
$$

so $c \geq 0$.

Suppose that $c>0$. If $\lim _{k \rightarrow \infty}\left\|u_{k}\right\|_{H^{\prime}(\Omega)}=0$, then for large $k, I\left(u_{k}\right)=o(1)$. Hence contradiction with the assumption that $c>0$. Thus there exist a subsequence, still denoted by $\left\{u_{k}\right\}$, a constant $c^{\prime}>0$, such that $\left\|u_{k}\right\|_{H^{1}(\Omega)} \geq c^{\prime}$.

LEMMA 2.2. For any $u \in M(\Omega)$, there exists a constant $K>0$ such that $I(u) \geq$ $((\theta-2) /(2 \theta))(2 K)^{(-2) /(p-1)}>0$.

Proof. By (f1) and (f2), for any $\varepsilon>0$ there exists a constant $C_{\varepsilon}>0$ such that

$$
|f(x, u)| \leq \varepsilon|u|+C_{\varepsilon}|u|^{p} .
$$

We take $\varepsilon=1 / 2$, and by the Sobolev inequality,

$$
\begin{aligned}
0 & =\left(I^{\prime}(u), u\right)=\|u\|_{H^{1}(\Omega)}^{2}-\int_{\Omega} f(x, u) u d x \\
& \geq\|u\|_{H^{1}(\Omega)}^{2}-\int_{\Omega}\left(\frac{1}{2} u^{2}+C_{1 / 2}|u|^{p+1}\right) d x \\
& \geq \frac{1}{2}\|u\|_{H^{1}(\Omega)}^{2}-K\|u\|_{H^{1}(\Omega)}^{p+1}=\|u\|_{H^{1}(\Omega)}^{2}\left(\frac{1}{2}-K\|u\|_{H^{1}(\Omega)}^{p-1}\right),
\end{aligned}
$$

thus $\|u\|_{H^{1}(\Omega)} \geq(2 K)^{(-1) /(p-1)}$, and then by $(\mathrm{f} 3)$,

$$
\begin{aligned}
I(u) & =\frac{1}{2}\|u\|_{H^{\prime}(\Omega)}^{2}-\int_{\Omega} F(x, u) d x \geq \frac{1}{2}\|u\|_{H^{1}(\Omega)}^{2}-\frac{1}{\theta} \int_{\Omega} f(x, u) u d x \\
& =\left(\frac{1}{2}-\frac{1}{\theta}\right)\|u\|_{H^{1}(\Omega)}^{2} \geq \frac{\theta-2}{2 \theta}(2 K)^{-2 /(p-1)} .
\end{aligned}
$$

Notice that $\delta(\Omega)$, the infimum of all positive $(P S)$-values, is a positive number. This can be proved as follows. By Stuart [8], $\alpha_{M}(\Omega)$ is a positive $(P S)_{\alpha_{M}(\Omega)}$-value, so $\Theta(\Omega)$ is not empty and $\delta(\Omega) \leq \alpha_{M}(\Omega)$.

For an arbitrary sequence $\left\{u_{k}\right\}$ bounded in $L^{2}\left(\mathbb{R}^{N}\right)$, we introduce the concentration functions of $\left|u_{k}\right|^{2}$,

$$
\Phi_{k}(t)=\sup _{z \in \mathbb{R}^{N}} \int_{B_{t}^{N}(z)}\left|u_{k}\right|^{2}
$$

defined for $t \geq 0$. 
LEMMA 2.3. Let $\left\{u_{k}\right\}$ be bounded in $H^{1}\left(\mathbb{R}^{N}\right)$ and assume that for some $t_{0}>0$, $\Phi_{k}\left(t_{0}\right) \rightarrow 0$. Then $u_{k} \rightarrow 0$ strongly in $L^{q}\left(\mathbb{R}^{N}\right)$ for all $2<q<2^{*}=2 N /(N-2)$. If in addition $u_{k}$ satisfies $\left(I^{\prime}\left(u_{k}\right), u_{k}\right) \rightarrow 0$, then $u_{k} \rightarrow 0$ strongly in $H^{1}\left(\mathbb{R}^{N}\right)$.

Proof. We divide the proof into two steps.

Step 1. Decompose $\mathbb{R}^{N}$ into unit cubes $F_{0}=\left\{P_{i}^{1}\right\}_{i=1}^{\infty}$ of length 1 with vertex at lattice points. Continuing to bisect the cubes to obtain cubes $F_{m}=\left\{P_{i}^{m}\right\}_{i=1}^{\infty}$ of length $1 / 2^{m}$ for each $P_{i}^{m}$. Let $m_{0}$ satisfy $\sqrt{N}\left(1 / 2^{m_{0}}\right)<t_{0}$. For each $i$, let $B_{i}^{m_{0}}$ be a ball with center at the same as that of $P_{i}^{m_{0}}$ and of radius $t_{0}$ in $\mathbb{R}^{N}$. Then $P_{i}^{m_{0}} \subset B_{i}^{m_{0}}, \mathbb{R}^{N}=\bigcup_{i=1}^{\infty} P_{i}^{m_{0}}$, and $\left\{P_{i}^{m_{0}}\right\}_{i=1}^{\infty}$ are nonoverlapping. Write $P_{i}=P_{i}^{m_{0}}$. If we take $q$ and $r$ such that $2<q<r<2^{*}$, we can write, using the Hölder inequality and Sobolev imbedding,

$$
\begin{aligned}
\int_{\mathbb{R}^{N}}\left|u_{k}\right|^{q} & =\sum_{i=1}^{\infty} \int_{P_{i}}\left|u_{k}\right|^{q}=\sum_{i=1}^{\infty} \int_{P_{i}}\left|u_{k}\right|^{2(r-q) /(r-2)}\left|u_{k}\right|^{r(q-2) /(r-2)} \\
& \leq \sum_{i=1}^{\infty}\left(\int_{P_{i}}\left|u_{k}\right|^{2}\right)^{(r-q) /(r-2)}\left(\int_{P_{t}}\left|u_{k}\right|^{r}\right)^{(q-2) /(r-2)} \\
& \leq\left(\Phi_{k}\left(t_{0}\right)\right)^{(r-q) /(r-2)} \sum_{i=1}^{\infty}\left(\int_{P_{i}}\left|u_{k}\right|^{r}\right)^{(q-2) /(r-2)} \\
& \leq c\left(\Phi_{k}\left(t_{0}\right)\right)^{(r-q) /(r-2)} \sum_{i=1}^{\infty}\left(\int_{P_{i}}\left(\left|\nabla u_{k}\right|^{2}+u_{k}^{2}\right)\right)^{r(q-2) / 2(r-2)}
\end{aligned}
$$

Since $\lim _{r \rightarrow q}(r(q-2) / 2(r-2))=q / 2>1$, we may choose $r$ such that $s=$ $(r(q-2) / 2(r-2)) \geq 1$.

$$
\begin{aligned}
\sum_{i=1}^{\infty}\left(\int_{P_{i}}\left(\left|\nabla u_{k}\right|^{2}+\left|u_{k}\right|^{2}\right)\right)^{r(q-2) / 2(r-2)} & =\sum_{i=1}^{\infty}\left(\int_{P_{i}}\left(\left|\nabla u_{k}\right|^{2}+\left|u_{k}\right|^{2}\right)\right)^{s} \\
& \leq\left(\sum_{i=1}^{\infty} \int_{P_{i}}\left(\left|\nabla u_{k}\right|^{2}+\left|u_{k}\right|^{2}\right)\right)^{s} \\
& =\left(\int_{\mathbb{R}^{N}}\left(\left|\nabla u_{k}\right|^{2}+\left|u_{k}\right|^{2}\right)\right)^{s} \\
& =\left\|u_{k}\right\|_{H^{1}\left(\mathbb{R}^{N}\right)}^{2 s} \leq c .
\end{aligned}
$$

Therefore, $u_{k} \rightarrow 0$ strongly in $L^{q}\left(\mathbb{R}^{N}\right)$ for all $2<q<2^{*}=2 N /(N-2)$.

Step 2. If, in addition, $u_{k}$ satisfies $\left(I^{\prime}\left(u_{k}\right), u_{k}\right) \rightarrow 0$, then for large $k,\left\|u_{k}\right\|_{H^{1}\left(\mathbb{R}^{N}\right)}^{2}=$ $\int_{\mathbb{R}^{N}} f\left(x, u_{k}\right) u_{k} d x+o(1)$. By (2.1), if $k$ is large,

$$
\begin{aligned}
\left\|u_{k}\right\|_{H^{1}\left(\mathbb{R}^{N}\right)}^{2} & \leq \int_{\mathbb{R}^{N}}\left|f\left(x, u_{k}\right)\right|\left|u_{k}\right| d x+o(1) \\
& \leq \varepsilon\left\|u_{k}\right\|_{H^{1}\left(\mathbb{R}^{N}\right)}^{2}+C_{\varepsilon}\left\|u_{k}\right\|_{L^{p+1}\left(\mathbb{R}^{N}\right)}^{p+1}+o(1),
\end{aligned}
$$


or by Step 1,

$$
(1-\varepsilon)\left\|u_{k}\right\|_{H^{1}\left(\mathbb{R}^{N}\right)}^{2} \leq C_{\varepsilon}\left\|u_{k}\right\|_{L^{p+1}\left(\mathbb{R}^{N}\right)}^{p+1}+o(1)=o(1) .
$$

Hence $u_{k} \rightarrow 0$ strongly in $H^{1}\left(\mathbb{R}^{N}\right)$.

LEMMA 2.4. Let $\left\{u_{k}\right\}$ be a $(P S)_{c}$-sequence with $c>0$. Then there exists a sequence $\left\{t_{k}\right\}$ in $\mathbb{R}_{+}$such that $\left\{t_{k} u_{k}\right\} \subset M(\Omega),\left\{t_{k}\right\}$ is bounded, and for large $k, \alpha_{M}(\Omega) \leq$ $I\left(t_{k} u_{k}\right) \leq c+o(1)$.

PROOF. Let $\left\{u_{k}\right\}$ be a $(P S)_{c}$-sequence with $c>0$, thus for large $k, u_{k} \nless 0$, and $u_{k} \nrightarrow 0$ strongly in $H^{1}\left(\mathbb{R}^{N}\right)$, where $u_{k}$ is identified with an element of $H^{1}\left(\mathbb{R}^{N}\right)$ by extending $u_{k}$ to be zero on $\mathbb{R}^{N} \backslash \Omega$, then by Lemma 2.3 , there exist a sequence $\left\{z_{k}\right\} \subset \mathbb{R}^{N}$ and $\varepsilon_{1}>0$ such that $u_{k} \Varangle 0$ in $B_{1 / 2}^{N}\left(z_{k}\right)$, and

$$
\int_{B_{1 / 2}^{N}\left(z_{k}\right)}\left|u_{k}(x)\right|^{2} d x \geq \varepsilon_{1}
$$

Hence there exist $\varepsilon_{2}>0, \varepsilon_{3}>0$, such that

$$
\left|D_{k}\right| \equiv\left|\left\{x \in B_{1 / 2}^{N}\left(z_{k}\right) \mid u_{k}(x) \geq \varepsilon_{2}\right\}\right| \geq \varepsilon_{3},
$$

where $\left|D_{k}\right|$ denotes the Lebesgue measure of the set $D_{k}$.

For $u_{k} \not \equiv 0$, by (f4), there exists a unique positive number $t_{k}$ such that $t_{k} u_{k} \in M(\Omega)$, then

$$
\left\|u_{k}\right\|_{H^{1}(\Omega)}^{2}=\int_{\Omega} \frac{f\left(x, t_{k} u_{k}\right) u_{k}}{t_{k}} d x
$$

Either $t_{k} \leq 1$ or $t_{k}>1$ in which case by (f3), $F(x, t y) / t^{\theta}$ is a nondecreasing function of $t>0$ for all $x \in \Omega$ and $y \in \mathbb{R} \backslash\{0\}$, then

$$
t_{k}^{2}\left\|u_{k}\right\|_{H^{\prime}(\Omega)}^{2}=\int_{\Omega} f\left(x, t_{k} u_{k}\right) t_{k} u_{k} d x \geq \theta \int_{\Omega} F\left(x, t_{k} u_{k}\right) d x \geq \theta \int_{\Omega} t_{k}^{\theta} F\left(x, u_{k}\right) d x .
$$

Consequently by Lemma 2.1 ,

$$
\begin{aligned}
t_{k}^{\theta-2} & \leq \theta^{-1} \frac{\left\|u_{k}\right\|_{H^{\prime}(\Omega)}^{2}}{\int_{\Omega} F\left(x, u_{k}\right) d x} \leq \theta^{-1} \frac{\bar{c}^{2}}{\int_{D_{k}} F\left(x, u_{k}\right) d x} \\
& \leq \theta^{-1} \frac{\bar{c}^{2}}{\int_{D_{k}} F\left(x, \varepsilon_{2}\right) d x} \leq \theta^{-1} \frac{\bar{c}^{2}}{\varepsilon_{3}\left(\min _{x \in \overline{D_{k}}} F\left(x, \varepsilon_{2}\right)\right)}
\end{aligned}
$$


thus $\left\{t_{k}\right\}$ must be bounded.

$$
\begin{aligned}
I\left(t_{k} u_{k}\right) & -I\left(u_{k}\right) \\
= & \frac{1}{2} t_{k}^{2}\left\|u_{k}\right\|_{H^{\prime}(\Omega)}^{2}-\int_{\Omega} F\left(x, t_{k} u_{k}\right) d x-\frac{1}{2}\left\|u_{k}\right\|_{H^{1}(\Omega)}^{2}+\int_{\Omega} F\left(x, u_{k}\right) d x \\
= & \frac{1}{2}\left(t_{k}^{2}-1\right) \int_{\Omega} f\left(x, u_{k}\right) u_{k} d x-\int_{\Omega} F\left(x, t_{k} u_{k}\right) d x+\int_{\Omega} F\left(x, u_{k}\right) d x+o(1) \\
= & g\left(t_{k}\right)+o(1)
\end{aligned}
$$

where $g(t)=\left(\left(t^{2}-1\right) / 2\right) \int_{\Omega} f\left(x, u_{k}\right) u_{k} d x-\int_{\Omega} F\left(x, t u_{k}\right) d x+\int_{\Omega} F\left(x, u_{k}\right) d x$. Since

$$
\begin{aligned}
g^{\prime}(t) & =t \int_{\Omega} f\left(x, u_{k}\right) u_{k} d x-\int_{\Omega} f\left(x, t u_{k}\right) u_{k} d x \\
& =t\left(\int_{\Omega} \frac{f\left(x, u_{k}\right) u_{k}}{1} d x-\int_{\Omega} \frac{f\left(x, t u_{k}\right) u_{k}}{t} d x\right),
\end{aligned}
$$

it follows from (f4) that $g^{\prime}(t)>0$ if $t \in(0,1)$ and $g^{\prime}(t)<0$ if $t \in(1, \infty)$. Thus $g(1)=\max _{t \in[0, \infty)} g(t), g(1)=0$, and for large $k$,

$$
I\left(t_{k} u_{k}\right)-I\left(u_{k}\right)=g\left(t_{k}\right)+o(1) \leq g(1)+o(1)=o(1) .
$$

Hence if $k$ is large, $\alpha_{M}(\Omega) \leq I\left(t_{k} u_{k}\right) \leq c+o(1)$.

Next, we prove that an optimal lower bound for $\Theta(\Omega)$ is $\alpha_{M}(\Omega)$ when (f1)-(f4) are satisfied.

THEOREM 2.5. If (f1)-(f4) hold, then $\delta(\Omega)=\alpha_{M}(\Omega)$.

PROOF. It suffices to show $\delta(\Omega) \geq \alpha_{M}(\Omega)$, since the reversed inequality is always true. Let $\left\{u_{k}\right\}$ be a $(P S)_{c}$-sequence with $c>0$, and by Lemma 2.4 , there exists $t_{k} \in$ $(0, \infty)$, such that $t_{k} u_{k} \in M(\Omega),\left\{t_{k}\right\}$ is bounded, and for large $k, \alpha_{M}(\Omega) \leq I\left(t_{k} u_{k}\right) \leq$ $c+o(1)$. Since $c$ is arbitrary positive (PS)-value, it follows that $\alpha_{M}(\Omega) \leq \delta(\Omega)$.

\section{Existence of solutions}

In this section, we show that if $\alpha_{M}(\Omega)<\alpha_{M}\left(\widetilde{\Omega}_{k}\right)$ for some large $k \in \mathbb{N}$, then there exists a ground state solution $u$ of (1.1) with $I(u)=\alpha_{M}(\Omega)$, and if there exists a $(P S)_{c}$-sequence with $\alpha_{M}(\Omega)<c<\alpha_{M}\left(\widetilde{\Omega}_{k}\right)$ for some large $k \in \mathbb{N}$, then there exist at least two positive solutions of (1.1), that is, a ground state solution $u$ and a positive higher energy solution.

First, we state some properties of Palais-Smale sequences. 
LEMMA 3.1. Let $\left\{u_{k}\right\}$ be a $(P S)$-sequence for I satisfying $u_{k} \rightarrow u$ weakly in $H_{0}^{1}(\Omega)$. Then

(1) $u$ is a weak solution of (1.1).

(2) If $u \neq \equiv$, then $u$ is a positive solution of (1.1).

(3) If $\left\{u_{k}\right\}$ is a $(P S)_{\alpha_{M}(\Omega)}$-sequence for I satisfying $u_{k} \rightarrow u$ weakly in $H_{0}^{1}(\Omega)$ and $u$ $\not \equiv$, then $u_{k} \rightarrow u$ strongly in $H_{0}^{1}(\Omega)$.

PROOF. (1) Take a subsequence $\left\{u_{k}\right\}$ such that $u_{k} \rightarrow u$ weakly in $H_{0}^{1}(\Omega)$, a.e. in $\Omega$, and strongly in $L_{l o c}^{q}(\Omega)$ for $1 \leq q<2^{*}$. Given $\phi \in C_{c}^{\infty}(\Omega)$, we get

$$
\int_{\Omega} \nabla u_{k} \cdot \nabla \phi \rightarrow \int_{\Omega} \nabla u \cdot \nabla \phi, \quad \int_{\Omega} u_{k} \phi \rightarrow \int_{\Omega} u \phi,
$$

and by (f2), $\left|f\left(x, u_{k}\right)-f(x, u)\right||\phi| \leq a_{1}\left(\left|u_{k}\right|+\left|u_{k}\right|^{p}+|u|+|u|^{p}\right)|\phi|$, then by the generalization of the Lebesgue dominated convergence theorem, we have

$$
\int_{\Omega} f\left(x, u_{k}\right) \phi \rightarrow \int_{\Omega} f(x, u) \phi .
$$

Hence $\left(I^{\prime}(u), \phi\right)=\lim _{k \rightarrow \infty}\left(I^{\prime}\left(u_{k}\right), \phi\right)=0$. Since $C_{c}^{\infty}(\Omega)$ is dense in $H_{0}^{1}(\Omega)$, we have $I^{\prime}(u)=0$. Therefore $u$ is a weak solution of (1.1).

(2) If $u$ is a nonzero solution of (1.1), then $u \in M(\Omega)$. By elliptic regularity, any critical point of $I$ is a classical solution of $(1.1)$. Let $u^{-}(x)=\max (-u(x), 0)$. Since

$$
0=\left(I^{\prime}(u), u^{-}\right)=\int_{\Omega} \nabla u \cdot \nabla u^{-}+\int_{\Omega} u u^{-}-\int_{\Omega} f(x, u) u^{-}=-\left\|u^{-}\right\|_{H^{1}(\Omega)}^{2},
$$

hence $u \geq 0$. By the maximum principle, $u>0$ in $\Omega$.

(3) By Part (2), $u \in M(\Omega)$ and applying Fatou's lemma yields

$$
\begin{aligned}
\alpha_{M}(\Omega) & \leq I(u)=\frac{1}{2}\|u\|_{H^{1}(\Omega)}^{2}-\int_{\Omega} F(x, u) d x \\
& =\frac{1}{2} \int_{\Omega} f(x, u) u d x-\int_{\Omega} F(x, u) d x \\
& \leq \liminf _{k \rightarrow \infty} \int_{\Omega}\left(\frac{1}{2} f\left(x, u_{k}\right) u_{k}-F\left(x, u_{k}\right)\right) d x=\lim _{k \rightarrow \infty} I\left(u_{k}\right)=\alpha_{M}(\Omega),
\end{aligned}
$$

or

$$
I(u)=\alpha_{M}(\Omega) .
$$

Set $p_{k}=u_{k}-u$ to get $p_{k} \rightarrow 0$ weakly in $H_{0}^{1}(\Omega)$, a.e. in $\Omega$, and strongly in $L_{\text {loc }}^{q}(\Omega)$ where $1 \leq q<2^{*}$, then for large $k$, we have

$$
\left\|p_{k}\right\|_{H^{\prime}(\Omega)}^{2}=\left\|u_{k}\right\|_{H^{\prime}(\Omega)}^{2}-\|u\|_{H^{\prime}(\Omega)}^{2}+o(1) .
$$


Since $u \in H_{0}^{1}(\Omega)$, by (f1) and (f2), for any $\varepsilon>0$, there exists $r>0$ such that for $\widetilde{\Omega}_{r}=\Omega \backslash \widehat{B_{r}^{N}(0)}$,

$$
\int_{\tilde{\Omega}_{r}}|u|^{2}<\varepsilon, \quad \int_{\tilde{\Omega}_{r}}|u|^{p+1}<\varepsilon, \quad \int_{\tilde{\Omega}_{r}}|F(x, u)|<\varepsilon .
$$

By the generalization of the Lebesgue dominated convergence theorem, we have

$$
\int_{\Omega_{r}} F\left(x, u_{k}\right) \rightarrow \int_{\Omega_{r}} F(x, u) \text { and } \int_{\Omega_{r}} F\left(x, p_{k}\right) \rightarrow 0
$$

Then

$$
\int_{\Omega_{r}}\left|F\left(x, p_{k}\right)-F\left(x, u_{k}\right)+F(x, u)\right|<\varepsilon
$$

Now by the Hölder inequality and the fact that $\left\|p_{k}\right\|_{H^{1}(\Omega)}$ and $\left\|u_{k}\right\|_{H^{\prime}(\Omega)}$ are bounded, we have

$$
\begin{aligned}
& \int_{\tilde{\Omega}_{r}}\left|F\left(x, p_{k}\right)-F\left(x, u_{k}\right)\right| \\
&=\int_{\widetilde{\Omega}_{r}}\left|f\left(x, t p_{k}+(1-t) u_{k}\right) \| u\right| \quad \text { for some } 0<t<1 \\
& \leq c \int_{\widetilde{\Omega}_{r}}\left(\left|p_{k}\right|+\left|u_{k}\right|+\left|p_{k}\right|^{p}+\left|u_{k}\right|^{p}\right)|u| \\
& \leq c\left(\left\|p_{k}\right\|_{H^{1}(\Omega)}\|u\|_{L^{2}\left(\tilde{\Omega}_{r}\right)}+\left\|u_{k}\right\|_{H^{1}(\Omega)}\|u\|_{L^{2}\left(\tilde{\Omega}_{r}\right)}\right. \\
&\left.+\left\|p_{k}\right\|_{H^{1}(\Omega)}^{p}\|u\|_{L^{p+1}\left(\tilde{\Omega}_{r}\right)}+\left\|u_{k}\right\|_{H^{1}(\Omega)}^{p}\|u\|_{L^{p+1}\left(\tilde{\Omega}_{r}\right)}\right) \\
&<\varepsilon .
\end{aligned}
$$

Therefore by (3.3), (3.4), and (3.5), for large $k$,

$$
\int_{\Omega} F\left(x, p_{k}\right)=\int_{\Omega} F\left(x, u_{k}\right)-\int_{\Omega} F(x, u)+o(1) .
$$

By (3.1), (3.2), and (3.6), for large $k$,

$$
I\left(p_{k}\right)=I\left(u_{k}\right)-I(u)+o(1)=\alpha_{M}(\Omega)-\alpha_{M}(\Omega)+o(1)=o(1),
$$

and it follows that

$$
\left\|p_{k}\right\|_{H^{\prime}(\Omega)}^{2}=2 \int_{\Omega} F\left(x, p_{k}\right) d x .
$$

For $\phi \in C_{c}^{\infty}(\Omega)$, by (f2), $\left|f\left(x, u_{k}-u\right) \phi\right| \leq a_{1}\left(\left|u_{k}-u\right|+\left|u_{k}-u\right|^{p}\right)|\phi|$, then by the generalization of the Lebesgue dominated convergence theorem again, we have 
$\int_{\Omega} f\left(x, u_{k}-u\right) \phi \rightarrow 0$. Since $C_{c}^{\infty}(\Omega)$ is dense in $H_{0}^{1}(\Omega), f\left(x, u_{k}-u\right) \rightarrow 0$ in $H^{-1}(\Omega)$. Similarly, $f\left(x, u_{k}\right)-f(x, u) \rightarrow 0$ in $H^{-1}(\Omega)$. So for large $k$, we have

$$
\begin{aligned}
I^{\prime}\left(p_{k}\right)= & -\Delta p_{k}+p_{k}-f\left(x, p_{k}\right)=-\Delta\left(u_{k}-u\right)+\left(u_{k}-u\right)-f\left(x, u_{k}-u\right) \\
= & \left(-\Delta u_{k}+u_{k}-f\left(x, u_{k}\right)\right)-(-\Delta u+u-f(x, u)) \\
& -\left(f\left(x, u_{k}-u\right)-f\left(x, u_{k}\right)+f(x, u)\right) \\
= & I^{\prime}\left(u_{k}\right)-I^{\prime}(u)+o(1)=o(1)
\end{aligned}
$$

and it follows that

$$
\left\|p_{k}\right\|_{H^{\prime}(\Omega)}^{2}=\int_{\Omega} f\left(x, p_{k}\right) p_{k} d x .
$$

From (f3), (3.7), and (3.8), for large $k$, we have $\left\|p_{k}\right\|_{H^{1}(\Omega)}=o(1)$, that is, $u_{k} \rightarrow u$ strongly in $H_{0}^{1}(\Omega)$.

We shall see what will happen when $u$ is zero. Let

$$
\Omega_{k}=\Omega \cap B_{k}^{N}(0), \quad \widetilde{\Omega}_{k}=\Omega \backslash \overline{B_{k}^{N}(0)} .
$$

LEMMA 3.2. Let $\left\{u_{k}\right\}$ be a $(P S)_{c}$-sequence with $c>0$. Then

(1) If (f1)-(f3) hold, suppose that $u_{k} \rightarrow 0$ weakly in $H_{0}^{1}(\Omega)$, then there exists a subsequence $\left\{u_{k}\right\}$ such that for $\Omega_{2 k}$, if $k$ is large,

$$
\int_{\Omega_{2 k}}\left|u_{k}\right|^{q}=o(1) \quad \text { for each } 1 \leq q<2^{*} .
$$

(2) In addition to (f1)-(f3), that (f4) satisfied, suppose there exists a subsequence, still denoted by $\left\{u_{k}\right\}$, such that for $\Omega_{2 k}$, if $k$ is large,

$$
\int_{\Omega_{2 k}}\left|u_{k}\right|^{q}=o(1) \quad \text { for each } 1 \leq q<2^{*} .
$$

Then we have $c \geq \alpha_{M}\left(\widetilde{\Omega}_{k}\right)$ for all large $k$.

(3) If (f1)-(f4) hold, suppose that $u_{k} \rightarrow 0$ weakly in $H_{0}^{1}(\Omega)$, then $c \geq \alpha_{M}\left(\widetilde{\Omega}_{k}\right)$ for all large $k$.

PROOF. (1) Since $u_{k} \rightarrow 0$ weakly in $H_{0}^{1}(\Omega)$, a.e. in $\Omega$, and strongly in $L_{\text {loc }}^{q}(\Omega)$, where $1 \leq q<2^{*}$. Thus for each $m \in \mathbb{N}, \lim _{k \rightarrow \infty} \int_{\Omega_{m}}\left|u_{k}\right|^{q}=0$. We can take a subsequence $\left\{u_{k_{m}}\right\}$ such that $\int_{\Omega_{m}}\left|u_{k_{m}}\right|^{q}<1 / m$. Therefore, there exists a subsequence, still denoted by $\left\{u_{k}\right\}$, such that for $\Omega_{2 k}$, if $k$ is large, $\int_{\Omega_{2 k}}\left|u_{k}\right|^{q}=o(1)$ for each $1 \leq q<2^{*}$. 
(2) Let $\left\{u_{k}\right\}$ be a $(P S)_{c}$-sequence, so for large $k$,

$$
\begin{gathered}
I\left(u_{k}\right)=\frac{1}{2}\left\|u_{k}\right\|_{H^{1}(\Omega)}^{2}-\int_{\Omega} F\left(x, u_{k}\right) d x=c+o(1), \\
\left\|u_{k}\right\|_{H^{1}(\Omega)}^{2}=\int_{\Omega} f\left(x, u_{k}\right) u_{k} d x+o(1) .
\end{gathered}
$$

Let $\zeta \in C^{\infty}([0, \infty))$ such that

$$
0 \leq \zeta \leq 1, \quad \zeta(t)= \begin{cases}0 & \text { for } t \in[0,1] \\ 1 & \text { for } t \in[2, \infty)\end{cases}
$$

Let $\zeta_{k}(x)=\zeta(|x| / k)$. Since $\left\{\zeta_{k}^{2} u_{k}\right\}$ is bounded in $H_{0}^{1}(\Omega)$, for large $k$,

$$
\begin{aligned}
o(1) & =\left(I^{\prime}\left(u_{k}\right), \zeta_{k}^{2} u_{k}\right) \\
& =\int_{\Omega}\left(\zeta_{k}^{2}\left|\nabla u_{k}\right|^{2}+2 \zeta_{k} u_{k} \nabla \zeta_{k} \cdot \nabla u_{k}+\zeta_{k}^{2} u_{k}^{2}\right) d x-\int_{\Omega} f\left(x, u_{k}\right) \zeta_{k}^{2} u_{k} d x
\end{aligned}
$$

Note that $\left|\nabla \zeta_{k}(x)\right| \leq c / k$, if $k$ is large, $\int_{\Omega_{2 k}}\left|u_{k}\right|^{q}=o(1)$ for each $1 \leq q<2^{*}$, so for large $k$,

$$
\int_{\Omega} \zeta_{k} u_{k} \nabla \zeta_{k} \cdot \nabla u_{k}=o(1)
$$

and by (f2), $\int_{\Omega_{2 k}} f\left(x, u_{k}\right) u_{k} d x=o(1)$. Then we have

$$
\int_{\Omega} f\left(x, u_{k}\right) \zeta_{k}^{2} u_{k} d x=\int_{\Omega} f\left(x, u_{k}\right) u_{k} d x+o(1)=\left\|u_{k}\right\|_{H^{1}(\Omega)}^{2}+o(1)
$$

again by (f2),

$$
\int_{\Omega} f\left(x, \zeta_{k} u_{k}\right) \zeta_{k} u_{k} d x=\int_{\Omega} f\left(x, u_{k}\right) \zeta_{k} u_{k} d x+o(1)=\left\|u_{k}\right\|_{H^{1}(\Omega)}^{2}+o(1)
$$

and

$$
\begin{aligned}
\int_{\Omega} F\left(x, \zeta_{k} u_{k}\right) d x \\
\quad=\int_{\Omega}\left(F\left(x, \zeta_{k} u_{k}\right)-F\left(x, u_{k}\right)\right) d x+\int_{\Omega} F\left(x, u_{k}\right) d x \\
\quad=\int_{\Omega_{2 k}} f\left(x,(1-t) u_{k}+t \zeta_{k} u_{k}\right)\left(\zeta_{k} u_{k}-u_{k}\right) d x+\int_{\Omega} F\left(x, u_{k}\right) d x \\
\quad=\int_{\Omega} F\left(x, u_{k}\right) d x+o(1), \quad \text { where } 0<t<1 .
\end{aligned}
$$


For large $k$, substituting (3.10), (3.11) into (3.9) yields

$$
\int_{\Omega} \zeta_{k}^{2}\left(\left|\nabla u_{k}\right|^{2}+u_{k}^{2}\right)=\left\|u_{k}\right\|_{H^{\prime}(\Omega)}^{2}+o(1) .
$$

Then by (3.13) and (3.14), for large $k$,

$$
\begin{aligned}
I\left(\zeta_{k} u_{k}\right)= & \frac{1}{2} \int_{\Omega}\left[\left|\nabla \zeta_{k}\right|^{2} u_{k}^{2}+\zeta_{k}^{2}\left(\left|\nabla u_{k}\right|^{2}+u_{k}^{2}\right)+2 \zeta_{k} u_{k} \nabla \zeta_{k} \cdot \nabla u_{k}\right] \\
& -\int_{\Omega} F\left(x, \zeta_{k} u_{k}\right) d x \\
= & \frac{1}{2}\left\|u_{k}\right\|_{H^{\prime}(\Omega)}^{2}-\int_{\Omega} F\left(x, u_{k}\right) d x+o(1) \\
= & I\left(u_{k}\right)+o(1)=c+o(1) .
\end{aligned}
$$

By (3.14), (3.12), for large $k$,

$$
\begin{aligned}
\left(I^{\prime}\left(\zeta_{k} u_{k}\right), \zeta_{k} u_{k}\right) & =\left\|\zeta_{k} u_{k}\right\|_{H^{1}(\Omega)}^{2}-\int_{\Omega} f\left(x, \zeta_{k} u_{k}\right) \zeta_{k} u_{k} d x \\
& =\left\|u_{k}\right\|_{H^{1}(\Omega)}^{2}-\left\|u_{k}\right\|_{H^{1}(\Omega)}^{2}+o(1)=o(1) .
\end{aligned}
$$

Let $v_{k}=\zeta_{k} u_{k} \in H_{0}^{1}\left(\widetilde{\Omega}_{k}\right)$. For $v_{k} \not \equiv 0$, by (3.15), (3.16) and Lemma 2.4, there exists $t_{k} \in(0, \infty)$ such that $t_{k} v_{k} \in M\left(\widetilde{\Omega}_{k}\right),\left\{t_{k}\right\}$ is bounded, and for large $k, \alpha_{M}\left(\widetilde{\Omega}_{k}\right) \leq$ $I\left(t_{k} v_{k}\right) \leq I\left(v_{k}\right)+o(1)=c+o(1)$. So we have $\alpha_{M}\left(\widetilde{\Omega}_{k}\right) \leq c$ for all large $k$.

(3) It follows immediately from Step (1) and Step (2).

Now we will prove the existence of a ground state solution and a positive higher energy solution of (1.1).

THEOREM 3.3. Suppose (f1)-(f4) hold, there exists a ground state solution $u$ of (1.1) with $I(u)=\alpha_{M}(\Omega)$ if $\alpha_{M}(\Omega)<\alpha_{M}\left(\widetilde{\Omega}_{k}\right)$ for some large $k \in \mathbb{N}$.

PrOOF. As a consequence of Ekeland's variational principle, there exists a sequence $\left\{u_{k}\right\} \subset M(\Omega)$ which weakly converges to $u$, such that $\left\{u_{k}\right\}$ is a $(P S)_{\alpha_{M}(\Omega)}$-sequence. If $\alpha_{M}(\Omega)<\alpha_{M}\left(\widetilde{\Omega}_{k}\right)$ for some large $k \in \mathbb{N}$, by Lemma 3.2 , replacing $c$ by $\alpha_{M}(\Omega), u \neq \equiv$, and then by Lemma 3.1, $u>0, u_{k} \rightarrow u$ strongly in $H_{0}^{1}(\Omega)$, and $I(u)=\alpha_{M}(\Omega)$.

THEOREM 3.4. If (f1)-(f4) hold, suppose there exists a $(P S)_{c}$-sequence with $\alpha_{M}(\Omega)<c<\alpha_{M}\left(\widetilde{\Omega}_{k}\right)$ for some large $k \in \mathbb{N}$, then there exists a positive higher energy solution $v$ of $(1.1)$ with $c \geq I(v)>\alpha_{M}(\Omega)$.

PROOF. Let $\left\{v_{k}\right\} \subset H_{0}^{1}(\Omega)$ be a $(P S)_{c}$-sequence with $\alpha_{M}(\Omega)<c<\alpha_{M}\left(\widetilde{\Omega}_{k}\right)$ for some large $k \in \mathbb{N}$. Take a subsequence $\left\{v_{k}\right\}$ such that $v_{k} \rightarrow v$ weakly in $H_{0}^{1}(\Omega)$, a.e. 
in $\Omega$, and strongly in $L_{\text {loc }}^{q}(\Omega)$ where $1 \leq q<2^{*}$. By Lemma $3.2, v \not \equiv 0$, then by Lemma 3.1, $v$ is a positive solution of (1.1) with $c \geq I(v) \geq \alpha_{M}(\Omega)$.

Suppose $I(v)=\alpha_{M}(\Omega)$. From Theorem 3.3, $I(u)=\alpha_{M}(\Omega)$. Setting $w_{k}=v_{k}-v$ and following the same line of proof as in Lemma 3.1 (3), for large $k$, we have

$$
\begin{aligned}
I\left(w_{k}\right) & =I\left(v_{k}\right)-I(v)+o(1)=c-\alpha_{M}(\Omega)+o(1), \\
I^{\prime}\left(w_{k}\right) & =I^{\prime}\left(v_{k}\right)-I^{\prime}(v)+o(1)=o(1),
\end{aligned}
$$

so $\left\{w_{k}\right\}$ is a $(P S)_{c-\alpha_{M}(\Omega)}$-sequence. Since $0<c-\alpha_{M}(\Omega)<\alpha_{M}\left(\widetilde{\Omega}_{k}\right)$ for some large $k \in \mathbb{N}$, by Lemma $3.2(3)$, it follows that $w_{k} \rightarrow w \neq \equiv 0$, a contradiction.

\section{The (PS)-conditions}

Let $\Lambda_{2}$ be a smooth domain in $\mathbb{R}^{N}$ and $\Lambda_{1}$ be a closed subset of $\Lambda_{2}$, then the relation between $\alpha_{M}\left(\Lambda_{2}\right)$ and $\alpha_{M}\left(\Lambda_{1}\right)$ is given by the following theorem.

THEOREM 4.1. Let $\Lambda_{1} \subset \Lambda_{2}$. If the functional I satisfies the $(P S)_{\alpha_{M}\left(\Lambda_{1}\right)}$-condition, then $\alpha_{M}\left(\Lambda_{2}\right)<\alpha_{M}\left(\Lambda_{1}\right)$.

PRoOF. $\Lambda_{1} \subset \Lambda_{2}$, so $\alpha_{M}\left(\Lambda_{2}\right) \leq \alpha_{M}\left(\Lambda_{1}\right)$. Suppose $\alpha_{M}\left(\Lambda_{2}\right)=\alpha_{M}\left(\Lambda_{1}\right)$. As a consequence of Ekeland's variational principle, there exists a sequence $\left\{u_{k}\right\} \subset M\left(\Lambda_{1}\right)$ such that $I\left(u_{k}\right) \rightarrow \alpha_{M}\left(\Lambda_{1}\right), I^{\prime}\left(u_{k}\right) \rightarrow 0$ in $H^{-1}\left(\Lambda_{1}\right)$. Since $I$ satisfies the $(P S)_{\alpha_{M}\left(\Lambda_{1}\right)^{-}}$ condition, there exist a subsequence $\left\{u_{k}\right\}$, and $u \in H_{0}^{1}\left(\Lambda_{1}\right)$, satisfying $u_{k} \rightarrow u$ strongly in $H_{0}^{1}\left(\Lambda_{1}\right)$. Since $\alpha_{M}\left(\Lambda_{1}\right)>0, u \neq \equiv 0$, then by Lemma $3.1, u>0$ with $I(u)=\alpha_{M}\left(\Lambda_{1}\right), I^{\prime}(u)=0$. And $I(u)=\alpha_{M}\left(\Lambda_{2}\right)=\inf _{u \in M\left(\Lambda_{2}\right)} I(u)$. It is known that every minimizer of $\alpha_{M}\left(\Lambda_{2}\right)$ is a critical point of $I$, therefore $u$ solves (1.1) if $\Omega=\Lambda_{2}$. By Lemma 3.1, $u>0$ in $\Lambda_{2}$. This contradicts $u \in H_{0}^{1}\left(\Lambda_{1}\right)$. Therefore, $\alpha_{M}\left(\Lambda_{2}\right)<\alpha_{M}\left(\Lambda_{1}\right)$.

Then we can verify that the $(P S)_{\alpha_{M}(\Omega)}$-condition is satisfied.

THEOREM 4.2. If (f1)-(f4) hold, then I satisfies the $(P S)_{\alpha_{M}(\Omega)}$-condition if and only if $\alpha_{M}(\Omega)<\lim _{k \rightarrow \infty} \alpha_{M}\left(\widetilde{\Omega}_{k}\right)$.

Proof. (1) Suppose $\alpha_{M}(\Omega)<\lim _{k \rightarrow \infty} \alpha_{M}\left(\widetilde{\Omega}_{k}\right)$, then $\alpha_{M}(\Omega)<\alpha_{M}\left(\widetilde{\Omega}_{k}\right)$ for some large $k \in \mathbb{N}$. Let $\left\{u_{k}\right\}$ be a $(P S)_{\alpha_{M}(\Omega)}$-sequence satisfying $u_{k} \rightarrow u$ weakly in $H_{0}^{1}(\Omega)$. By Lemma 3.2, $u \neq \equiv 0$, then by Lemma 3.1, $u_{k} \rightarrow u$ strongly in $H_{0}^{1}(\Omega)$. We conclude that $I$ satisfies the $(P S)_{\alpha_{M}(\Omega)}$-condition.

(2) We argue indirectly. Suppose $\alpha_{M}(\Omega)=\lim _{k \rightarrow \infty} \alpha_{M}\left(\widetilde{\Omega}_{k}\right)$, then $\alpha_{M}(\Omega)=$ $\alpha_{M}\left(\widetilde{\Omega}_{k}\right)$ for all $k \in \mathbb{N}$. We claim that $I$ does not satisfy the $(P S)_{\alpha_{M}(\Omega)}$-condition in $\Omega$. 
In fact, suppose on the contrary, $I$ satisfies the $(P S)_{\alpha_{M}(\Omega)}$-condition in $\Omega$. Then we claim that $\left.I\right|_{H_{0}^{1}\left(\tilde{\Omega}_{k}\right)}$ satisfies the $(P S)_{\alpha_{M}(\Omega)}$-condition in $\widetilde{\Omega}_{k}$ for all $k \in \mathbb{N}$. In fact, let $\left\{u_{n}\right\} \subset H_{0}^{1}\left(\widetilde{\Omega}_{k}\right) \subset H_{0}^{1}(\Omega)$ satisfy $I\left(u_{n}\right) \rightarrow \alpha_{M}\left(\tilde{\Omega}_{k}\right), I^{\prime}\left(u_{n}\right) \rightarrow 0$ in $H^{-1}\left(\widetilde{\Omega}_{k}\right)$. Since $I$ satisfies the $(P S)_{\alpha_{M}(\Omega)}$-condition in $\Omega$, there exist a subsequence $\left\{u_{n}\right\}$, and $u \in H_{0}^{1}(\Omega)$ satisfying $u_{n} \rightarrow u$ strongly in $H_{0}^{1}(\Omega)$, that is, $u_{k} \rightarrow u$ strongly in $H_{0}^{1}\left(\widetilde{\Omega}_{k}\right)$. Therefore $\left.I\right|_{H_{0}^{1}\left(\tilde{\Omega}_{k}\right)}$ satisfies the $(P S)_{\alpha_{M}\left(\tilde{\Omega}_{k}\right)}$-condition. By Theorem 4.1, $\alpha_{M}(\Omega)<\alpha_{M}\left(\widetilde{\Omega}_{k}\right)$. This is a contradiction.

\section{Upper half strip with a hole}

In this section, the domain is the upper half strip with a hole. For simplicity, we consider the case where $f$ does not depend on $x$, so the problem is as follows:

$$
\begin{cases}-\Delta u+u=f(u) & \text { in } \Lambda, \\ u>0 & \text { in } \Lambda, \\ u \in H_{0}^{1}(\Lambda), & \end{cases}
$$

where $\Lambda=A_{0}^{r} \backslash D, D \subset B_{\rho}^{N}\left(\left(0, \eta_{0}\right)\right) \subset A_{0}^{r}$, and $\eta_{0}$ is sufficiently large, $\rho$ is sufficiently small, and they are suitably chosen. Let

$$
\begin{gathered}
Q_{s}\left(\eta_{0}\right)=\left\{(\xi, \eta) \in \mathbb{R}^{N-1} \times \mathbb{R}|| \xi|<r, \quad| \eta-\eta_{0} \mid<s\right\}, \\
\tilde{Q}_{s}\left(\eta_{0}\right)=A_{0}^{r} \backslash Q_{s}\left(\eta_{0}\right), \quad \Lambda_{m}=\Lambda \cap Q_{m}\left(\eta_{0}\right), \quad \tilde{\Lambda}_{m}=\Lambda \backslash \overline{Q_{m}\left(\eta_{0}\right)} .
\end{gathered}
$$

THEOREM 5.1. (1) $\alpha_{M}(\Lambda)=\alpha_{M}\left(A^{r}\right)$.

(2) The functional I does not satisfy the $(P S)_{\alpha_{M}(\Lambda)}$-condition, and the only possible solutions of (5.1) are higher energy solutions.

PROOF. (1) Let $w \in H_{0}^{1}\left(A^{r}\right)$ be the positive solution of (5.1) if $\Lambda=A^{r}$ with $I(w)=\alpha_{M}\left(A^{r}\right)$. Take $\left\{\left(0, \eta_{n}\right)\right\} \subset \Lambda, r_{n} \rightarrow \infty$ such that $Q_{r_{n}}\left(\eta_{n}\right) \subset \Lambda$. Consider the cut-off function $\psi \in C_{c}^{\infty}([0, \infty))$ such that

$$
0 \leq \psi \leq 1, \quad \psi(t)= \begin{cases}1 & \text { for } t \in[0,1] \\ 0 & \text { for } t \in[2, \infty)\end{cases}
$$

Let $w_{n}(\xi, \eta)=\psi\left(2\left|\eta-\eta_{n}\right| / r_{n}\right) w\left(\xi, \eta-\eta_{n}\right)$. Then $w_{n} \in H_{0}^{1}(\Lambda)$. Since for large $k$,

$$
\begin{aligned}
\left\|w_{n}(\xi, \eta)-w\left(\xi, \eta-\eta_{n}\right)\right\|_{H^{\prime}\left(A^{\prime}\right)}^{2} & \\
\quad & \left\|\psi\left(2\left|\eta-\eta_{n}\right| / r_{n}\right) w\left(\xi, \eta-\eta_{n}\right)-w\left(\xi, \eta-\eta_{n}\right)\right\|_{H^{\prime}\left(A^{\prime}\right)}^{2} \\
& \leq \int_{A^{r} \cap \bar{Q}_{n_{n} / 2\left(\eta_{n}\right)}}\left(\left|\nabla w\left(\xi, \eta-\eta_{n}\right)\right|^{2}+w\left(\xi, \eta-\eta_{n}\right)^{2}\right)+o(1) \\
& =o(1),
\end{aligned}
$$


and by (f2), for large $k$,

$$
\begin{aligned}
& \int_{A^{\prime}} \mid F\left(w_{n}(\xi, \eta)\right)-F\left(w\left(\xi, \eta-\eta_{n}\right)\right) \mid \\
&= \int_{A^{\prime} \cap \tilde{Q}_{m^{\prime} / 2\left(\eta_{n}\right)}}\left|f\left(t w_{n}(\xi, \eta)+(1-t) w\left(\xi, \eta-\eta_{n}\right)\right)\right|\left|w_{n}(\xi, \eta)-w\left(\xi, \eta-\eta_{n}\right)\right| \\
& \leq c \int_{A^{r} \cap \tilde{Q}_{m^{\prime} / 2\left(\eta_{n}\right)}}\left(\left|w\left(\xi, \eta-\eta_{n}\right)\right|^{2}+\left|w\left(\xi, \eta-\eta_{n}\right)\right|^{p+1}\right) \\
&= o(1), \quad \text { for some } 0<t<1, \\
& \int_{A^{\prime}}\left|f\left(w_{n}(\xi, \eta)\right) w_{n}(\xi, \eta)-f\left(w\left(\xi, \eta-\eta_{n}\right)\right) w\left(\xi, \eta-\eta_{n}\right)\right| \\
& \leq \int_{A^{\prime}}\left|f\left(w_{n}(\xi, \eta)\right) w_{n}(\xi, \eta)-f\left(w_{n}(\xi, \eta)\right) w\left(\xi, \eta-\eta_{n}\right)\right| \\
& \quad+\int_{A^{r}}\left|f\left(w_{n}(\xi, \eta)\right) w\left(\xi, \eta-\eta_{n}\right)-f\left(w\left(\xi, \eta-\eta_{n}\right)\right) w\left(\xi, \eta-\eta_{n}\right)\right| \\
&=
\end{aligned}
$$

then we have

$$
\begin{aligned}
I\left(w_{n}\right) & =\frac{1}{2}\left\|w_{n}\right\|_{H^{1}(\Lambda)}^{2}-\int_{\Lambda} F\left(w_{n}\right) \\
& =I(w)+o(1)=\alpha_{M}\left(A^{r}\right)+o(1), \\
\left(I^{\prime}\left(w_{n}\right), w_{n}\right) & =\left\|w_{n}\right\|_{H^{1}(\Lambda)}^{2}-\int_{\Omega} f\left(w_{n}\right) w_{n} \\
& =\|w\|_{H^{1}\left(A^{\prime}\right)}^{2}-\int_{A^{r}} f(w) w+o(1)=o(1) .
\end{aligned}
$$

For $w_{n} \in H_{0}^{1}(\Lambda), w_{n} \not \equiv 0$, by (5.2), (5.3), and Lemma 2.4, there exists $t_{n} \in(0, \infty)$ such that $t_{n} w_{n} \in M(\Lambda),\left\{t_{n}\right\}$ is bounded, and $\alpha_{M}(\Lambda) \leq I\left(t_{n} w_{n}\right) \leq I\left(w_{n}\right)+o(1)=$ $\alpha_{M}\left(A^{r}\right)+o(1)$ for large $n$. Hence we obtain $\alpha_{M}(\Lambda)=\alpha_{M}\left(A^{r}\right)$.

(2) By Part 1 and Theorem 4.1, $I$ does not satisfy the $(P S)_{\alpha_{M}(\Lambda)}$-condition. If $u$ is a ground state solution of (5.1), by putting $u=0$ in $A^{r} \backslash \Lambda$, we see that $u$ could be regarded as an element of $H_{0}^{1}\left(A^{r}\right)$; then by the strong maximum principle, $u$ would be a positive solution in $A^{r}$, a contradiction. Therefore the only possible solutions of (5.1) are positive higher energy solutions.

Using the same argument as in the proof of Theorem 5.1, we obtain

PROPOSITION 5.2. (1) Let $\Sigma$ be a closed subset of $A^{r}$. If for any $s>0$ there exists $(0, \eta) \in \Sigma$ such that $Q_{s}(\eta) \subset \Sigma$, then $\alpha_{M}(\Sigma)=\alpha_{M}\left(A^{r}\right)$. As a more concrete example, $\Sigma$ can be a upper half strip $A_{0}^{r}$, a upper half strip with a hole $\Lambda$ or the union of $A_{0}^{r}$ with a bounded set. 
(2) $\lim _{\eta \rightarrow \infty} \alpha_{M}\left(Q_{\eta}(\eta)\right)=\alpha_{M}\left(A_{0}^{r}\right)=\alpha_{M}\left(A^{r}\right)=\alpha_{M}\left(A_{a}^{r}\right)$, for every $a \in \mathbb{R}$.

From Theorem 5.1 and Proposition 5.2, we know that there doesn't exist any $(P S)_{c}$ sequence with $\alpha_{M}(\Lambda)<c<\alpha_{M}\left(\tilde{\Lambda}_{k}\right)$ for any $k \in \mathbb{N}$, so we cannot use Theorem 3.4 to get the existence of any higher energy solution of (5.1). Hence we take a new approach to obtain a higher energy solution of (5.1).

THEOREM 5.3. If (f1)-(f4) hold, suppose there exists a $(P S)_{c}$-sequence with $c>0$ and $c \notin \Theta\left(\tilde{\Lambda}_{m}\right)$ for some $m \in \mathbb{N}$, then there exists a positive higher energy solution of (5.1).

PROOF. Let $\left\{u_{k}\right\}$ be a $(P S)_{c}$-sequence with $c>0$ and $c \notin \Theta\left(\tilde{\Lambda}_{m}\right)$ for some $m \in \mathbb{N}$. Take a subsequence $\left\{u_{k}\right\}$ such that $u_{k} \rightarrow u$ weakly in $H_{0}^{1}(\Lambda)$, a.e. in $\Lambda$, and strongly in $L_{l o c}^{q}(\Lambda)$, where $1 \leq q<2^{*}$. Moreover, $I^{\prime}(u)=0$ and $I(u) \leq c$. We claim that $u \not \equiv 0$. Suppose $u \equiv 0$, as in the proof of Lemma 3.2, there exists a subsequence $\left\{u_{k}\right\}$ such that for $Q_{k}\left(\eta_{0}\right)$, if $k$ is large,

$$
\int_{Q_{k}\left(\eta_{0}\right)}\left|u_{k}\right|^{q}=o(1) \quad \text { for each } 1 \leq q<2^{*} .
$$

Let $\xi: \mathbb{R}^{N} \rightarrow[0,1]$ be a $C^{\infty}$-function which satisfies

$$
\xi(x)= \begin{cases}0 & \text { for } x \in B_{m}^{N}\left(0, \eta_{0}\right) \\ 1 & \text { for } x \notin B_{m+1}^{N}\left(0, \eta_{0}\right) .\end{cases}
$$

Let $w_{k}=\xi u_{k}, w_{k} \in H_{0}^{1}\left(\tilde{\Lambda}_{m}\right)$. Then we want to show that $\left\{w_{k}\right\}$ is a $(P S)_{c}$-sequence in $H_{0}^{1}\left(\tilde{\Lambda}_{m}\right)$.

It suffices to show that

$$
\lim _{k \rightarrow \infty}\left|I\left(w_{k}\right)-I\left(u_{k}\right)\right|=0
$$

and

$$
\lim _{k \rightarrow \infty} \sup _{\|\phi\|_{H^{\prime}\left(\dot{\lambda}_{m}\right)} \leq 1}\left|\left(I^{\prime}\left(w_{k}\right), \phi\right)-\left(I^{\prime}\left(u_{k}\right), \phi\right)\right|=0
$$

By a direct computation,

$$
\begin{aligned}
& \left|\left(I^{\prime}\left(w_{k}\right), \phi\right)-\left(I^{\prime}\left(u_{k}\right), \phi\right)\right| \\
& \leq\left|\int_{\tilde{\Lambda}_{m}}(\xi(x)-1) u_{k} \phi\right|+\left|\int_{\tilde{\Lambda}_{m}}(\xi(x)-1) \nabla u_{k} \cdot \nabla \phi\right| \\
& \quad+\left|\int_{\tilde{\Lambda}_{m}} u_{k} \nabla \xi \cdot \nabla \phi\right|+\left|\int_{\bar{\Lambda}_{m}}\left(f\left(u_{k}\right)-f\left(\xi u_{k}\right)\right) \phi\right|
\end{aligned}
$$




$$
\begin{aligned}
& \leq\left(\int_{\bar{\Lambda}_{m} \cap B_{m+1}^{N}\left(0, \eta_{0}\right)}\left|u_{k}\right|^{2}\right)^{1 / 2}+\left(\int_{\tilde{\Lambda}_{m} \cap B_{m+1}^{N}\left(0, \eta_{0}\right)}\left|\nabla u_{k}\right|^{2}\right)^{1 / 2} \\
& +\|\nabla \xi\|_{L^{\infty}}\left(\int_{\tilde{\Lambda}_{m} \cap B_{m+1}^{N}\left(0, \eta_{0}\right)}\left|u_{k}\right|^{2}\right)^{1 / 2} \\
& +2 a_{1}\left[\left(\int_{\bar{\Lambda}_{m} \cap B_{m+1}^{N}\left(0, \eta_{0}\right)}\left|u_{k}\right|^{2}\right)^{1 / 2}+\left(\int_{\tilde{\Lambda}_{m} \cap B_{m+1}^{N}\left(0, \eta_{0}\right)}\left|u_{k}\right|^{2 p}\right)^{1 / 2}\right]
\end{aligned}
$$

Since $\tilde{\Lambda}_{m} \bigcap B_{m+1}^{N}\left(0, \eta_{0}\right) \subset \Lambda_{k-1}$ if $k$ is large, (5.6) follows from (5.7) and (5.4), provided that

$$
\int_{\bar{\Lambda}_{m} \cap B_{m+1}^{N}\left(0, \eta_{0}\right)}\left|\nabla u_{k}\right|^{2} \rightarrow 0 \text { as } k \rightarrow \infty .
$$

Now we prove (5.8). Let $\xi_{k}: \mathbb{R}^{N} \rightarrow[0,1]$ be a $C_{0}^{\infty}$-function which satisfies $0 \leq \xi_{k} \leq 1,\left|\nabla \xi_{k}\right| \leq 1$, and

$$
\xi_{k}(x)= \begin{cases}1 & \text { for } x \in B_{k-1}^{N}\left(0, \eta_{0}\right) \\ 0 & \text { for } x \notin B_{k}^{N}\left(0, \eta_{0}\right)\end{cases}
$$

Since $\left\{\xi_{k} u_{k}\right\}$ is bounded in $H_{0}^{1}(\Lambda)$, if $k$ is large,

$$
\begin{aligned}
o(1) & =\left(I^{\prime}\left(u_{k}\right), \xi_{k} u_{k}\right) \\
& =\int_{\Lambda_{k}} \xi_{k}\left|\nabla u_{k}\right|^{2}+\int_{\Lambda_{k}} u_{k} \nabla \xi_{k} \cdot \nabla u_{k}+\int_{\Lambda_{k}} \xi_{k} u_{k}^{2}-\int_{\Lambda_{k}} f\left(x, u_{k}\right) \xi_{k} u_{k} .
\end{aligned}
$$

By (5.4), we conclude that the last three integrals of (5.9) tend to zero as $k \rightarrow \infty$ and consequently

$$
\int_{\tilde{\Lambda}_{m} \cap B_{m+1}^{N}\left(0, \eta_{0}\right)}\left|\nabla u_{k}\right|^{2} \leq \int_{\Lambda_{k-1}}\left|\nabla u_{k}\right|^{2} \leq \int_{\Lambda_{k}} \xi_{k}\left|\nabla u_{k}\right|^{2} \rightarrow 0 \text { as } k \rightarrow \infty
$$

Observe that

$$
\begin{aligned}
I\left(w_{k}\right)-I\left(u_{k}\right)= & \frac{1}{2} \int_{\Lambda}\left[\left(\xi^{2}-1\right)\left(\left|\nabla u_{k}\right|^{2}+u_{k}^{2}\right)+|\nabla \xi|^{2}\left|u_{k}\right|^{2}+2 \xi u_{k} \nabla \xi \cdot \nabla u_{k}\right] \\
& -\int_{\Lambda}\left(F\left(w_{k}\right)-F\left(u_{k}\right)\right) .
\end{aligned}
$$

Thus (5.5) follows from several estimates which are similar to the above. Hence $c \in \Theta\left(\tilde{\Lambda}_{m}\right)$, this is contrary to the hypothesis, so there exists a positive higher energy solution $u$. 
Next, we will use a mini-max structure to obtain a positive higher energy solution of (5.1).

From Theorem 5.1 and Proposition 5.2, we have $\lim _{\eta \rightarrow \infty} \alpha_{M}\left(Q_{\eta}(\eta)\right)=\alpha_{M}(\Lambda)$, then for any $\varepsilon_{4}>0$, there exists $\bar{\eta}=\bar{\eta}\left(\varepsilon_{4}\right)$ sufficiently large such that

$$
\alpha_{M}\left(A_{a}^{r}\right)=\alpha_{M}(\Lambda)<\alpha_{M}\left(Q_{\bar{\eta}}(\bar{\eta})\right)<\alpha_{M}(\Lambda)+\varepsilon_{4}, \quad \text { for every } a \in \mathbb{R} .
$$

So we can choose $\eta_{0}=9 \bar{\eta} / 2$ and $D \subset B_{\rho}^{N}\left(\left(0, \eta_{0}\right)\right) \subset Q_{\bar{\eta} / 4}\left(\eta_{0}\right) \subset A_{4 \bar{\eta}}^{r}$, and then choose $z_{-} \in M\left(Q_{\bar{\eta}}(\bar{\eta})\right)$ and $z_{+} \in M\left(A_{7 \bar{\eta}}^{r}\right)$ such that $\max \left(I\left(z_{-}\right), I\left(z_{+}\right)\right)<\alpha_{M}(\Lambda)+\varepsilon_{4}$. Set

$$
\Gamma=\left\{\gamma \in C([0,1], M(\Lambda)) \mid \gamma(0)=z_{-} \text {and } \gamma(1)=z_{+}\right\}, \quad \mu=\inf _{\gamma \in \Gamma} \max _{\theta \in[0,1]} I(\gamma(\theta)) .
$$

We will show that there exists a $(P S)_{\mu}$-sequence with $\mu>\alpha_{M}(\Lambda)$, provided that $z_{+}$ and $z_{\text {- }}$ are suitably chosen. Let $\varphi$ be a $C^{\infty}$ function which satisfies $0 \leq \varphi \leq 1$, $|\nabla \varphi| \leq 2 / \tilde{\eta}, \varphi \equiv 0$ on $Q_{\tilde{\eta} / 2}\left(\eta_{0}\right)$ and $\varphi \equiv 1$ on $\tilde{Q}_{\tilde{\eta}}\left(\eta_{0}\right)$. Straightforward calculation, gives the following lemma:

LEMMA 5.4. For any $\varepsilon_{5} \in\left(0, \alpha_{M}(\Lambda) / 2\right)$, there exists $\delta=\delta\left(\varepsilon_{5}\right)>0$ such that if $u \in M(\Lambda)$ and $I(u)<\alpha_{M}(\Lambda)+\delta$ then $I\left(t_{\varphi u} \varphi u\right)<\alpha_{M}(\Lambda)+\varepsilon_{5}$, where $t_{\varphi u}>0$ and $t_{\varphi u} \varphi u \in M(\Lambda)$.

With Lemma 5.4, we want to show that $\mu>\alpha_{M}(\Lambda)$.

LEMMA 5.5. $\mu \geq \alpha_{M}(\Lambda)+\delta$, where $\delta$ is the number defined in Lemma 5.4.

Proof. Suppose $\mu<\alpha_{M}(\Lambda)+\delta$. From the definition of $\mu$, there exists a $\gamma_{0} \in \Gamma$ such that $\max _{\theta \in[0,1]} I\left(\gamma_{0}(\theta)\right)<\alpha_{M}(\Lambda)+\delta$. Let $\gamma(\theta)=t_{\varphi \gamma_{0}(\theta)} \varphi \gamma_{0}(\theta)$, it follows from Lemma 5.4 that $\gamma \in \Gamma$ and

$$
\max _{\theta \in[0,1]} I(\gamma(\theta))<\alpha_{M}(\Lambda)+\varepsilon_{5}<\frac{3}{2} \alpha_{M}(\Lambda) .
$$

By the definition of $\varphi, \gamma(\theta)=\gamma_{+}(\theta)+\gamma_{-}(\theta)$, where $\gamma_{+}(\theta) \in A_{7 \bar{\eta}}^{r}$ and $\gamma_{-}(\theta) \in Q_{\bar{\eta}}(\bar{\eta})$. We claim that

(5.11) there exists a $\theta_{0} \in(0,1)$ such that $\gamma_{+}\left(\theta_{0}\right) \in M(\Lambda)$ and $\gamma_{-}\left(\theta_{0}\right) \in M(\Lambda)$.

Assuming (5.11) for now, we obtain $I\left(\gamma\left(\theta_{0}\right)\right)=I\left(\gamma_{+}\left(\theta_{0}\right)\right)+I\left(\gamma_{-}\left(\theta_{0}\right)\right)>\alpha_{M}(\Lambda)+$ $\alpha_{M}(\Lambda)=2 \alpha_{M}(\Lambda)$, which contradicts (5.10).

It remains to show (5.11) to complete the proof. Since $\gamma_{+}(0)=0$ and $\gamma_{+}(1)=z_{+}$, there exists a $\theta_{1} \in(0,1)$ such that $I^{\prime}\left(\gamma_{+}\left(\theta_{1}\right)\right) \gamma_{+}\left(\theta_{1}\right)>0$. This together with $\gamma\left(\theta_{1}\right) \in$ $M(\Lambda)$ implies that $I^{\prime}\left(\gamma_{-}\left(\theta_{1}\right)\right) \gamma_{-}\left(\theta_{1}\right)<0$. Let

$$
\theta_{2}=\sup \left\{\theta \mid I^{\prime}\left(\gamma_{-}(\theta) \gamma_{-}(\theta)<0 \text { or } \gamma_{-}(\theta) \in M(\Lambda)\right\} .\right.
$$


Since $\gamma_{-}(1)=0$ and $\gamma_{-}(0)=z_{-}$, it follows that $\theta_{2} \in(0,1)$. Using $I \in C^{1}$ and $I^{\prime}\left(\gamma_{-}\left(\theta_{2}\right)\right) \gamma_{-}\left(\theta_{2}\right)=0$. Since $\gamma\left(\theta_{2}\right) \in M(\Lambda)$, it follows that $I^{\prime}\left(\gamma_{+}\left(\theta_{2}\right)\right) \gamma_{+}\left(\theta_{2}\right)=0$.

To complete the proof of (5.11), we need to show that $\gamma_{-}\left(\theta_{2}\right) \neq 0$ and $\gamma_{+}\left(\theta_{2}\right) \neq 0$. We argue indirectly. If $\gamma_{-}\left(\theta_{2}\right)=0$, then either $\gamma_{-}(\theta)=0$ for all $\theta \in\left(\theta_{2}, 1\right)$ or there exists a $\theta_{3} \in\left(\theta_{2}, 1\right)$ such that $I^{\prime}\left(\gamma_{-}\left(\theta_{3}\right)\right) \gamma_{-}\left(\theta_{3}\right)>0$. This contradicts (5.12). Suppose $\gamma_{+}\left(\theta_{2}\right)=0$. Then there exists a $\theta_{4} \in\left(\theta_{2}, 1\right)$ such that $I^{\prime}\left(\gamma_{+}\left(\theta_{4}\right)\right) \gamma_{+}\left(\theta_{4}\right)>0$. This together with $\gamma\left(\theta_{4}\right) \in M(\Lambda)$ yields $I^{\prime}\left(\gamma_{-}\left(\theta_{4}\right)\right) \gamma_{-}\left(\theta_{4}\right)<0$, which again violates (5.12). Thus the proof is complete.

Then we will show the existence of a Palais-Smale sequence with the (PS)-value $\mu$.

LEMMA 5.6. There exists a $(P S)_{\mu}$-sequence, where $\mu$ is the number defined in Lemma 5.4 .

PRoof. Suppose there does not exist a $(P S)_{\mu}$-sequence. Then there exist $b>0$ and $\hat{\varepsilon}>0$ such that $\left\|I^{\prime}(u)\right\| \geq b$ for all $u$ with $\mu-\hat{\varepsilon}<I(u) \leq \mu+\hat{\varepsilon}$. We may assume without loss of generality that $b<1$ and $\hat{\varepsilon}<(1 / 2)\left(\mu-\alpha_{M}(\Lambda)-(\delta / 4)\right)$, where $\delta$ is the number defined in Lemma 5.4. Let $Y_{1}=\left\{u \in M(\Lambda) \mid\left\|I^{\prime}(u)\right\| \leq b / 2\right.$ and $I(u) \leq 3 \mu / 2\}$ and $Y_{2}=\left\{u \in M(\Lambda) \mid\left\|I^{\prime}(u)\right\| \geq b\right.$ and $\left.I(u) \leq 3 \mu / 2\right\}$. Choose

$$
\varepsilon \in\left(0, \varepsilon_{1}\right), \quad \text { where } \quad \varepsilon_{1}=\min \left(\hat{\varepsilon}, b^{2} / 2, b / 4\right) .
$$

Let $Y_{3}=\{u \in M(\Lambda) \mid I(u) \leq \mu-\hat{\varepsilon}$ or $I(u) \geq \mu+\hat{\varepsilon}\}$ and $Y_{4}=\{u \in M(\Lambda) \mid \mu-\varepsilon \leq$ $I(u) \leq \mu+\varepsilon\}$. For $u \in M(\Lambda)$, set $g_{1}(u)=\left\|u-Y_{3}\right\| /\left(\left\|u-Y_{3}\right\|+\left\|u-Y_{4}\right\|\right)$ and $g_{2}(u)=\left\|u-Y_{1}\right\| /\left(\left\|u-Y_{1}\right\|+\left\|u-Y_{2}\right\|\right)$. Let $X(u)$ be a pseudo-gradient vector field for $I$ on $M(\Lambda)$ and

$$
W(u)=-g_{1}(u) g_{2}(u) h(\|X(u)\|) X(u),
$$

where $h(s)=1$ if $s \in[0,1]$ and $h(s)=1 / s$ if $s \geq 1$.

Consider the Cauchy problem:

$$
\frac{d \eta}{d t}=W(\eta), \quad \eta(0, u)=u
$$

The basic existence-uniqueness theorem for ordinary differential equations implies that, for each $u \in M(\Lambda),(5.15)$ has a unique solution $\eta(t, u)$ which is defined for $t$ in a maximal interval $[0, T(u)$ ). Moreover, since $\|W(u)\| \leq 1$ and $M(\Lambda)$ is a closed subset of $H_{0}^{1}(\Lambda)$, so $T(u)=+\infty$. Since

$$
\frac{d}{d t} I(\eta(t, u))=-I^{\prime}(\eta(t, u)) g_{1}(\eta(t, u)) g_{2}(\eta(t, u)) h(\|X(\eta(t, u))\|) X(\eta(t, u)) .
$$


Define $\tilde{I}^{a}=\{u \in M(\Lambda) \mid I(u) \leq a\}$. Since $I(\eta(t, u))$ is a non-increasing function of $t$, hence

$$
\eta\left(1, \tilde{I}^{\mu-\varepsilon}\right) \subset \tilde{I}^{\mu-\varepsilon}
$$

We claim that

$$
\eta\left(1, Y_{4}\right) \subset \tilde{I}^{\mu-\varepsilon} .
$$

Indeed, if there exists $u \in Y_{4}$ such that $\eta(1, u) \notin \tilde{I}^{\mu-\varepsilon}$, then, for all $t \in[0,1]$, $\eta(t, u) \in Y_{4}$. Consequently $g_{1}(\eta(t, u))=1$ and $g_{2}(\eta(t, u))=1$. If for some $t \in(0,1),\|X(\eta(t, u))\| \leq 1$, then $h(\|X(\eta(t, u))\|)=1$ and

$$
\frac{d}{d t} I(\eta(t, u)) \leq-\left\|I^{\prime}(\eta(t, u))\right\|^{2} \leq-b^{2} .
$$

On the other hand, if for some $t \in(0,1),\|X(\eta(t, u))\|>1$, then by the definition of pseudo-gradient vector field,

(5.19) $\frac{d}{d t} I(\eta(t, u)) \leq-\left\|I^{\prime}(\eta(t, u))\right\|^{2}\|X(\eta(t, u))\|^{-1} \leq-\frac{1}{2}\left\|I^{\prime}(\eta(t, u))\right\| \leq-\frac{b}{2}$.

Since $\eta(t, u) \in Y_{4}$ for all $t \in[0,1]$, by (5.18) and (5.19), we have

$$
2 \varepsilon \geq I(\eta(0, u))-I(\eta(1, u))=-\int_{0}^{1} \frac{d}{d t} I(\eta(t, u)) d t \geq \min \left(b / 2, b^{2}\right) .
$$

Since (5.20) is contrary to (5.13), we conclude that (5.17) must hold. Combining (5.16) and (5.17), we have

$$
\eta\left(1, \tilde{I}^{\mu+\varepsilon}\right) \subset \tilde{I}^{\mu-\varepsilon} .
$$

By the definition of $\mu$, there exists a $\gamma \in \Gamma$ such that $\max _{\theta \in[0,1]} I(\gamma(\theta))<\mu+\varepsilon$. Let $\gamma_{1}(\theta)=\eta(1, \gamma(\theta))$. It follows from (5.21) that

$$
\max _{\theta \in[0,1]} I(\gamma(\theta)) \leq \mu-\varepsilon .
$$

Since $g_{1}(u)=0$ if $u \in \tilde{I}^{\mu-\hat{\varepsilon}}$, it follows from (5.14) and (5.15) that $\eta(1, u)=u$ if $u \in \tilde{I}^{\mu-\hat{\varepsilon}}$. In particular, $\max \left(I\left(z_{+}\right), I\left(z_{-}\right)\right)<\alpha_{M}(\Omega)+(\delta / 4)$ implies $\gamma_{1}(0)=\gamma(0)$, $\gamma_{1}(1)=\gamma(1)$ and consequently $\gamma_{1} \in \Gamma$. But then (5.22) is contrary to the definition of $\mu$. The proof is complete.

We are now ready to prove the existence of a positive higher energy solution of (5.1).

THEOREM 5.7. Assume that (f1)-(f4) hold. If $\mu \notin \Theta\left(\tilde{\Lambda}_{m}\right)$ for some $m \in \mathbb{N}$, then there exists a positive higher energy solution of (5.1).

PROOF. By Lemma 5.5 and Lemma 5.6, there exists a $(P S)_{\mu}$-sequence with $\mu>\alpha_{M}(\Lambda)$, then by Theorem 5.3, we obtain a positive higher energy function of (5.1). 


\section{Acknowledgement}

The author is grateful to the referee and the editor whose valuable comments helped to improve the content of this paper.

\section{References}

[1] V. Benci and G. Cerami, 'Positive solutions of some nonlinear elliptic problems in exterior domains', Arch. Rational Mech. Anal. 99 (1987), 283-300.

[2] K. J. Chen, Semilinear elliptic equations in infinite strip domains and flask domains (Ph.D. Thesis, National Tsing Hua university, Hsinchu, Taiwan, R.O.C., 1999).

[3] K. J. Chen, K. C. Chen and H. C. Wang, 'Symmetry of positive solutions of semilinear elliptic equations in infinite strip domains', J. Differential Equations 148 (1998), 1-8.

[4] J. M. Coron, 'Topologie et cas limite des injections de Sobolev', C. R. Acad. Sci. Paris Ser. I Math. 299 (1984), 209-212.

[5] M. J. Esteban and P. L. Lions, 'Existence and non-existence results for semilinear elliptic problems in unbounded domains', Proc. Roy. Soc. Edinburgh 93A (1982), 1-12.

[6] P. L. Lions, 'The concentration-compactness principle in the calculus of variations. The locally compact case', Ann. Inst. H. Poincaré Anal. Non Linéaire 1 (1984), Part 1, 109-145, Part 2, 223283.

[7] S. I. Pohozaev, 'Eigenfunctions of the equation $\Delta u+\lambda f(u)=0$ ', Soviet Math. Dokl. 6 (1965), 1408-1411.

[8] C. A. Stuart, 'Bifurcation in $L^{p}\left(\mathbb{B}^{n}\right)$ for a semilinear elliptic equation', Proc. London Math. Soc. 45 (1982), 169-192.

Department of Applied Science

Chinese Naval Academy

669 Jiun Shiaw Road

Kaohsiung

Taiwan R.O.C.

e-mail: kuanju@mail.cna.edu.tw 